optimal health outcomes. Supportive services (eg, housing, food, counselling, addiction treatment) are increasingly conceptualised as critical components of care. Our study investigates social and clinical correlates of supportive service use across differing levels of engagement. Abstract P5-S6.22 figure 1 presents the conceptual model of the study.

Methods The Longitudinal Investigations into Supportive and Ancillary Health Services (LISA) is a cross-sectional cohort of HIVpositive persons on antiretroviral therapy in British Columbia, Canada. Interviewer-administered surveys collected information regarding sociodemographic factors, substance use, social support networks, and supportive services. Clinical variables were obtained through longitudinal linkages with the Drug Treatment Program at the BC Centre for Excellence in HIV/AIDS. Participants were stratified by level of service engagement (daily, weekly, and 1-3 months). Bivariate analysis and a logistic regression proportional odds model were used to identify variables significantly associated with supportive service use.

Results Among 915 participants, 742 (81\%) reported using supportive services, of which 344 were highly engaged, 280 moderately engaged, and 118 minimally engaged. Food programs, medication support, and counselling and social supports groups were services most accessed. Multivariate results demonstrated that those most engaged in supportive services were more likely to report poor social determinants of health such as low income [adjusted OR $(A O R)=1.81$ ]; not having completed high school ( $\mathrm{AOR}=1.97)$; unstable housing ( $\mathrm{AOR}=1.89$ ); and current illicit drug use $(A O R=1.60)$. After adjusting for social determinants, there were no significant differences in clinical measures across different levels of engagement with supportive services.

Conclusion High service use by those demonstrating social and clinical vulnerabilities reaffirms the need for continued expansion of supportive services to facilitate a more equitable distribution of health among persons living with HIV.

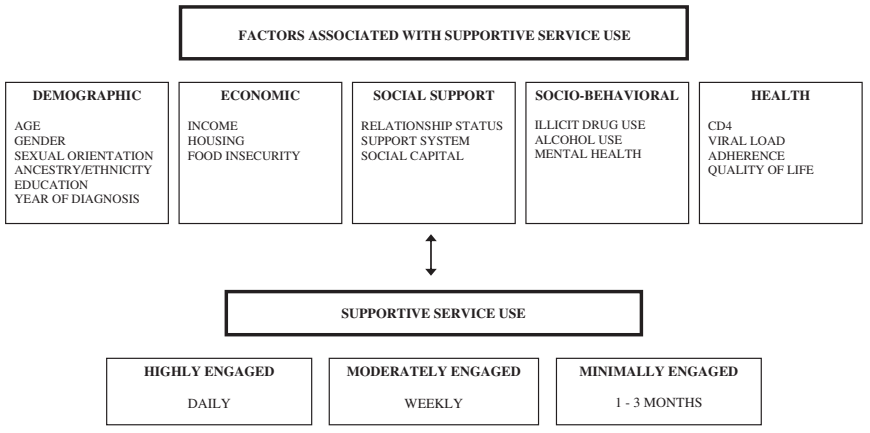

Abstract P5-S6.22 Figure 1 Conceptual model for factors associated with supportive service use.

\section{P5-S6.23 BARRIERS TO ACCESSING HEPATITIS C TREATMENT FOR INDIVIDUALS WHO HAVE EXPERIENCE WITH INJECTION DRUG USE}

doi:10.1136/sextrans-2011-050108.579

\section{Sinclair, S Kirkland, M Asbridge, F Martin. Dalhousie University, Halifax, Canada}

In Canada, approximately 10000 people are living with both hepatitis C and HIV; $20 \%$ of individuals living with HIV are coinfected with hepatitis C. Many individuals who inject drugs are at a higher risk for contracting both hepatitis $\mathrm{C}$ and HIV because they engage in high risk activities that increase their chances of being in contact with infected blood Individuals living with hepatitis $\mathrm{C}$ are at risk of contracting HIV. Being co-infected with both diseases complicates both hepatitis C and HIV; it is therefore critical to provide treatment for hepatitis C. Although those living with hepatitis C often report high interested in treatment, uptake remains low. The purpose of this research project is to identify the factors which influence decisions around hepatitis $C$ treatment. A mixed methods approach was used; 60 individuals participated in a cross sectional questionnaire, while 6 engaged in in-depth interviews. All participants were currently accessing methadone maintenance treatment for opioid addiction and had experience with injection drug use. The questionnaires explored characteristics, knowledge, attitude and willingness to access hepatitis $C$ treatment. Interviews delved deeper into the issues uncovered in the questionnaires and explored life experiences and their influence around treatment decisions. Results indicated that $70 \%$ of participants were interested in starting hepatitis $\mathrm{C}$ treatment within the next 6 months, while $30 \%$ were undecided or uninterested. Analysis of the questionnaire results have suggested that it may not be factual knowledge which influences individuals' decisions around treatment, but life conditions (ie, housing, employment) and experiences. The interviews supported this finding though a thematic analysis. The results of this study suggest that efforts to increase interest in treatment should focus on improving life conditions that support accessing treatment (eg, providing supportive housing). Future studies would include a larger sample size and a more refined questionnaire.

\section{P5-S6.24 COMPREHENSIVE, COMPETENT AND COMPASSIONATE CARE FOR PEOPLE LIVING WITH HIV IN COASTAL ANDHRA PRADESH}

doi:10.1136/sextrans-2011-050108.580

${ }^{1} \mathrm{R}$ Washington, ${ }^{2} \mathrm{R}$ Babu. ${ }^{1} \mathrm{KHPT}$, Bangalore, India; ${ }^{2}$ Lepra Society Secunderabad, India

Background Districts in coastal Andhra Pradesh, south India have the highest burden of HIV in both rural and urban areas. A comprehensive care program focused on improving clinical care and community outreach, complemented by a computerised management information system (CMIS), was implemented under the USAID supported Samastha project.

Methods Four community care centres covering 19 mandals were chosen as project sites. A mandal covers about 25 villages. Doctors, nurses and other members of the care teams were trained using the adapted "Integrated Management of Adult Illness" package and followed up with clinical mentorship visits every quarter. All on-site staffs were trained on infection prevention procedures. At the community level, people living with HIV (PLHIV) were involved in community outreach focused on treatment adherence, accompanied referrals for management of opportunistic infections and social linkages for livelihood options and basic nutrition. Small support groups of 10-12 individuals were formed at village or cluster level. Minor ailments and illnesses were treated at the local primary healthcare centers. Each community outreach worker maintained individual line-lists and prioritised outreach and follow-up for an average of 125 PLHIV, including children infected or affected by HIV. 6-8 outreach workers were supervised by a counsellor.

Results By the fourth year of implementation, 3257 PLHIV were registered with the project, of whom 1269 (38.9\%) were initiated on Government supplied free ART. 2845 (87.3\%) received nutrition support through education, supplementation and government schemes. 2085 (64\%) were linked to social schemes such as widow pensions, bank loans or alternative livelihood options. Psychosocial support was provided to 2964 (91\%) of the individuals registered. Loss to follow-up among those on ART reduced from $17 \%$ to $<1 \%$ and annual death rates among those registered decreased from $21 \%$ 